\section{Isolated short stature as a presentation of celiac disease in Saudi children}

\author{
Asaad Mohamed Abdullah Assiri \\ Department of Pediatrics, College of \\ Medicine and King Khalid University \\ Hospital, King Saud University, Riyadh, \\ Saudi Arabia
}

\section{Abstract}

The aim of this study is to assess the prevalence of isolated short stature as a clinical presentation of celiac disease in Saudi Arab children and whether some of the routine laboratory tests performed to determine the cause of short stature could suggest the diagnosis of celiac disease. A total of 91 children with short stature were included in the study. Extensive endocrine and biochemical assessments, including total protein, serum albumin, calcium phosphate and alkaline phosphatase assays; renal function tests; coagulation profile; anti-endomysial antibodies and anti-tissue transglutaminase antibody, growth hormone, thyroid stimulating hormone, free-thyroxin (FT4) assays; stool tests for giardiasis; bone age; and endoscopic intestinal biopsies, were done for all children. Ten of the 91 children had positive intestinal biopsies in the form of total villous atrophy, an increase in crypt height, and an increase in intra-epithelial lymphocyte (IEL) numbers up to >40 IEL/100 EC (Type 3C) according to the Oberhuber classification, confirming the diagnosis of celiac disease. Five children had mild villous atrophy according to this classification (Type 3A), and they were considered to have potential celiac disease. Seventy-six children had normal intestinal biopsies. Therefore, the prevalence of celiac disease among Saudi children with short stature was $10.9 \%$, and $4.3 \%$ of the children were diagnosed as having potential celiac disease. After confirming the diagnosis of celiac disease, all children were kept on a gluten-free diet and all of them showed improvement in their growth rate. We concluded that celiac disease is a very important cause of short stature in children without gastrointestinal complaints in Saudi Arabia. We highly recommend anti-tissue transglutaminase and anti-endomysial antibody screening tests, and a small bowel biopsy to confirm the diagnosis of celiac disease irrespective of the results of the antibody assays, in children with short stature in Saudi Arabia. Once the diagnosis is confirmed, children should be kept on a gluten-free diet so they can catch up their growth early before they develop permanent short stature.

\section{Introduction}

Celiac disease (CD) is a permanent intolerance to ingested gluten that results in immunologically mediated inflammatory damage to the small intestinal mucosa. CD must always be considered when dealing with children manifesting growth failure. The prevalence of CD among children with short stature in Europe is approximately $0.05-0.2 \%$ of the general population, reaching 1 in 300 in western Ireland. In Italy the prevalence is $59.1 \%^{1}$ whereas in Spain it is $0.56 \%{ }^{2}$ and in western New York 4.0\%. ${ }^{3}$ There has been no study conducted to assess its prevalence in children with short stature in Saudi Arabia.

Classically infants with CD present with impaired growth, diarrhea, and abdominal distension. Usually atypical presentation is seen in older children, who often have no overt features of malabsorption. ${ }^{4}$ Extra-intestinal manifestations such as short stature, delayed puberty, dental enamel defects, and arthralgia may occur as a monosymptomatic manifestation. ${ }^{5}$ The diagnosis of CD was based on clinical symptoms, positive antibodies, and a small bowel biopsy. ${ }^{6,7}$ Histological evidence of $\mathrm{CD}$ was reported according to the Oberhuber classification. ${ }^{8}$

Our aim in the present study is to assess the prevalence of CD among Saudi children with short stature who have no gastrointestinal symptoms, and to determine whether celiac serology can detect all cases of CD or if an intestinal biopsy should be performed for any case of short stature.

\section{Materials and Methods}

A total of 91 children with a height less than the $5^{\text {th }}$ centile adjusted for their age and gender were studied. All children were followed up in the Department of Pediatrics at King Khalid University Hospital from the period of August 2002 to December 2008. Any child with gastrointestinal symptoms was excluded from the study. All children with etiological factors known to produce growth failure had also been excluded; for example chromosomal anomalies, fetal growth failure, and chronic illnesses. Selection criteria are shown in Table 1.

All children had undergone an extensive endocrine work-up that included growth hormone $(\mathrm{GH})$, free-thyroxin $\left(\mathrm{FT}_{4}\right)$, thyroid stimulating hormone (TSH), glucose, electrolytes, venous blood gas, and urine $\mathrm{pH}$ assessments. The routine $\mathrm{GH}$ stimulation test using two biochemical (either clonidine and glucagon or insulin-induced hypoglycemia as a secretagogue) and two physiological (post-exercise and during sleep) assessments was performed.
Correspondence: Asaad Mohamed Abdullah Assiri, King Saud University, KKUH, Pediatric Department (39), P.0. Box 2925, Riyadh 11461, Kingdom of Saudi Arabia.

E-mail: prof.asaad@hotmail.com

Key words: celiac disease, short stature, anti-tissue transglutaminase, Saudi, children

Acknowledgements: the author would like to thank Ms. Loida M. Sese and Liza Antonio for their secretarial assistance.

Received for publication: 30 November 2009

Revision received: 14 February 2010.

Accepted for publication: 15 February 2010.

This work is licensed under a Creative Commons Attribution 3.0 License (by-nc 3.0).

(C) Copyright A.M.A. Assiri, 2010

Licensee PAGEPress, Italy

Pediatric Reports 2010; 2:e4

doi:10.4081/pr.2010.e4

Patients were considered not to be $\mathrm{GH}$ deficient when the peak $\mathrm{GH}$ value during the stimulation test or the physiological test was $>10$ $\mathrm{ng} / \mathrm{dL}$. Other routine investigations, such as hemoglobin level [MCV, MCHC, total protein, serum albumin, coagulation profile, calcium, phosphorous, and alkaline phosphatase (ALP)] were done. Stool investigations for giardiasis were done for all patients. Bone age was determined using the Greulich and Pyle atlas. ${ }^{9}$ Pubertal stages were evaluated according to Tanner. ${ }^{10}$ Mid-parental height was calculated for each child's parents to exclude genetic causes of short stature, using the standard formula. ${ }^{11}$

Anti-endomysial and anti-tissue transglutaminase screening tests for CD were performed for the patients. When no cause of short stature was found, upper digestive endoscopies and small bowel biopsies was done for all children. The biopsy specimens were taken from the third part of the duodenum.

The results of the children's heights before and after the gluten-free diet were expressed as a z-score.

\section{Results}

Ten of the ninety-one patients had a positive endoscopic intestinal biopsy with an increase in the crypt height and increase in intraepithelial lymphocyte (IEL) numbers up to $>40$ IEL/100EC, Type 3C, according to the Oberhuber classification, indicating CD (group 1; $\mathrm{N}=10$ ). Seventy-six children had a normal endoscopic intestinal biopsy, and five had mild villous atrophy with normal crypts, 
Type 3A according to this classification, and were considered to have potential CD (Group $2 ; \mathrm{N}=5$ ). Therefore, the prevalence of welldiagnosed CD among children of short stature in this study was $10.9 \%$ while $4.3 \%$ was diagnosed as having potential CD.

CD in our study affected six males and four females, thus the prevalence was equal in males and females. The mean values for chronological age, height, weight, bone age, as well as pubertal stage were not significantly different between patients with CD and nonceliacs $(\mathrm{P}>0.05)$. The results of serum calcium, phosphate, alkaline phosphatase, total protein, albumin assays, and hormonal tests were also not significantly different. Tests for celiac antibodies (anti-endomysial and antitissue transglutaminase) were done for all the patients and were strongly positive for ten children, who were confirmed histologically to have $\mathrm{CD}$, Type $3 \mathrm{C}$. Five patients were IgG positive for anti-endomysial antibody, shown histologically to be Type $3 \mathrm{~A}$.

All children diagnosed to have $\mathrm{CD}$ and potential CD were kept on a gluten-free diet. Patients were followed-up every six months and showed improvement in growth rate. Four of them had complete catch-up in growth after one year on a gluten-free diet (Table 2). The z-score for height before diagnosis was $2.14011 \pm 1.67487$ while it was $1.84149 \pm 1.83677$ one year after starting the gluten-free diet.

Table 2 shows age, gender, serology, histology, and height of children at the initial presentation and on follow-up of height one year on the gluten-free diet, as a z-score.

\section{Discussion}

This series shows a significant number of cases of CD and potential CD (10.9\% and 4.3\%) among Saudi children with short stature, who do not have underlying causes for their short statures. The prevalence of CD in children with short stature has been studied in different regions of the world. It ranged from $0.05-59.1 \%$ depending on the region of the study. ${ }^{1,3}$ In reviewing the literature we found no single parameter suggestive of CD in children with short-stature. ${ }^{12-15}$ This supports our data in this study.

None of the biochemical and hormonal measurements was positive in our patients. The pathogenesis of short stature as a monosymptomatic manifestation in children with $\mathrm{CD}$ is not known. A review of the literature showed that there are reasons to suggest that nutritional deficiencies can result in growth failure associated with changes in hormonal status. ${ }^{1}$ The hormonal abnormalities in these data included low levels of insulin-like growth factor-1 (IGF-I), which occurs after prolonged

Table 1. Selection criteria.

1 All short children, height $<5^{\text {th }}$ centile for age and gender

2 No gastrointestinal symptoms

3 Normal endocrine work-up (e.g. normal GH response, normal TFT)

$4 \quad$ No evidence of chronic disorders

5 Normal karyotype for female

6 Negative history of genetic short stature

Table 2. Age, gender, serology, histology, and height of children at the initial presentation and follow-up of height, one year on a gluten-free diet.

\begin{tabular}{lrrrrrrr}
\hline Age (years) & Gender & Serology & Histology & Ht 1 (cm) & Ht1 z-score Ht2 (cm) & Ht2 z-score \\
4.5 & M & ++ & Type 3C & 100.00 & -0.74439 & 114.50 & -0.15562 \\
11 & $\mathrm{~F}$ & ++ & Type 3C & 126.00 & 1.67487 & 134.00 & 1.83677 \\
\hline 6.5 & $\mathrm{M}$ & ++ & Type 3C & 106.00 & -0.18610 & 112.80 & -0.32931 \\
5 & $\mathrm{~F}$ & ++ & Type 3C & 98.00 & -0.93048 & 110.00 & -0.61540 \\
\hline 10 & $\mathrm{~F}$ & ++ & Type 3C & 117.00 & 0.83744 & 125.00 & 0.91721 \\
10 & $\mathrm{~F}$ & ++ & Type 3C & 117.00 & 0.83744 & 125.00 & 0.91721 \\
\hline 5 & $\mathrm{M}$ & ++ & Type 3C & 102.00 & -0.55829 & 125.00 & -0.61540 \\
4 & $\mathrm{M}$ & ++ & Type 3C & 102.00 & -0.55829 & 110.00 & -0.61540 \\
\hline 7 & $\mathrm{~F}$ & ++ & Type 3C & 85.00 & -2.14011 & 98.00 & -1.84149 \\
7 & $\mathrm{~F}$ & ++ & Type 3C & 107.00 & -0.09305 & 115.00 & -0.10453 \\
\hline 9 & $\mathrm{M}$ & + & Type 3A & 113.00 & 0.46524 & 120.00 & 0.40634 \\
6 & $\mathrm{~F}$ & + & Type 3A & 104.00 & -0.37219 & 110.00 & -0.61540 \\
\hline 8 & $\mathrm{M}$ & + & Type 3A & 110.00 & 0.18610 & 107.00 & -0.92192 \\
12 & $\mathrm{~F}$ & + & Type 3A & 119.00 & 1.02353 & 127.00 & 1.12155 \\
\hline 12 & $\mathrm{~F}$ & + & Type 3A & 119.00 & 1.02353 & 127.00 & 1.12155
\end{tabular}

$\begin{array}{lll}\text { Z-score } & 2.14011 \pm 1.67487 & 1.84149 \pm 1.83677\end{array}$

1. At the initial presentation. 2.Follow-up of height after one year on a gluten-free diet. Histology according to the Oberhuber classification ${ }^{8}$; $(+)$, positive; (-), negative; ++, anti-tissue transglutaminase; +, anti-endomysial $\lg \mathrm{G}$.

exposure to gluten, and poor growth hormone release in stimulatory tests. ${ }^{16}$ Other endocrine abnormalities that can occur in CD included secondary hypopituitarism probably caused by severe malnutrition and mainly found in longstanding CD, hypogonadism and delayed puberty, reversed insensitivity to androgens, elevated plasma testosterone, free testosterone index, and raised plasma luteinizing hormone. ${ }^{1,17}$ All children included in our study had no gastrointestinal symptoms or malnutrition and $\mathrm{GH}$ and thyroid hormone secretion were normal.

The ultimate adult height will improve if the treatment of $\mathrm{CD}$ is started early; however, there will be a slight negative effect of the disease on growth. ${ }^{18}$ Most of our children in this study who were followed up after one year showed an increase in their heights. Height and weight velocities had their maximum rates during the first year after initiation of the gluten-free diet, but the catch-up growth was incomplete over three years, the final height seemingly influenced mainly by familial characteristics. $^{18}$

We concluded that CD is prevalent among Saudi children with short stature. The fact that there was no difference between CD and non-
CD clinical, biochemical, and hormonal tests indicates that it is important to do antiendomysial and anti-tissue transglutaminase antibody tests to investigate all children with short stature for CD. In addition, it is important to do a small bowel biopsy for children with short stature, irrespective of the result of celiac serology, in Saudi Arabia and all over the world.

\section{References}

1. Bonamico M, Sciré G, Mariani P, et al. Short stature as the primary manifestation of monosymptomatic celiac disease. J Pediatr Gastroenterol Nutr 1992;14:12-6.

2. Corera Sanchez M, Vilate Carrasco A, Igea $\mathrm{J}$, et al. Celiac disease and short stature. An Esp Pediatr 1992;37:304-6.

3. Rossi TM, Albini CH, Kuma V. Incidence of celiac disease identified by the presence of serum endomysial antibiodies in children with chronic diarrhea, short stature in Insulin Dependent Diabetes. J Pediatr 1993;123:262-4.

4. Farrell RJ, Kelly CP. Celiac sprue. New Engl 
J Med 2002;346:180-8.

5. Verkasalo M, Kuitunen P, Leisti S, et al. Growth failure from symptomless celiac disease. A study of 14 patients. Helv Paediatr Acta 1978;33:489-95.

6. Anonymous. American Gastroenterological Association medical position statement: celiac sprue. Gastroenterology 2001;120: 1522-5.

7. Anonymous. Revised criteria for diagnosis of celiac disease. Report of Working Group of European Society of Paediatric Gastroenterology and Nutrition. Arch Dis Child 1990;65:909-11.

8. Oberhuber G, Granditsch G, Vogelsong H. The histology of celiac disease: time for a standardized report scheme for pathologists. Eur J Gastroenterol Hepatol 1999; 11:1185-94.

9. Greulich WW, Pyle SI. Radiographic atlas of skeletal development of the hand and wrist. 2nd edn, Stanford University Press: Stanford, California, 1959.

10. Tanner JM. Growth at adolescence with a general consideration of the effect of hereditary and environmental factors upon growth and maturation from birth to maturity. 2nd edn, Blackwell Scientific Publications: Oxford, 1962.

11. Wales JKH, Wit J-M, Rogol AD. Pediatric Endocrinology and Growth. 2nd edn, Saunders Scientific Publications: 0xford, 2002.

12. Groll A, Candy DC, Preece MA, et al. Short stature as the primary manifestation of celiac disease. Lancet 1980; 2:1097-9.

13. Knudtazon J, Fluge G, Aksnesl L. Routine measurements of gluten antibodies in children of short stature. J Pediatr Nutr 1991;12:190-4.

14. Bomanico M, Ballati G, Mariani P, et al. Screening for celiac disease: the meaning of low titre of anti-gliadin antibodies (AGA) in non-celiac children. Eur $\mathrm{J}$ Epidemiol 1997;13:55-9.

15. Eichler I, Frisch H, Granditsch G. Growth failure and skin insulin-like growth factor (IGF-I) in childhood celiac disease. Klinische Wochenschrift 1991;69:825-9.

16. Cacciari E, Salardi S, Lazzari R, et al. Short stature and celiac disease: a relationship to consider even in patients with no gastrointestinal tract symptoms. J Pediatr 1983;103:708-11.

17. Gemme G, Vingolo M, Naselli A, et al. Linear growth and skeletal maturation in subjects with treated celiac disease. J Pediatr Gastroenterol Nutr 1999;29:339-42.

18. Bosio L, Barrera G, Mistura L, et al. Growth acceleration and final height after treatment for delayed diagnosis of celiac disease. J Pediatr Gastroenterol Nutr 1990;11: 324-9. 